\title{
Higher doses of a green tea-based supplement increase post-exercise blood flow following an acute resistance exercise bout in recreationally resistance-trained college-aged men
}

\author{
Carlton D. Fox ${ }^{1}$, Christian T. Garner ${ }^{1}$, Petey W. Mumford ${ }^{2}$, Darren T. Beck ${ }^{1,3}$ and Michael D. Roberts ${ }^{1,3^{*}}$ (D)
}

\begin{abstract}
Background: There are animal data suggesting green tea can enhance blood flow. However, human data are lacking. Thus, the purpose of this study was to examine the acute effects of low and high doses of a green teabased supplement (GBS) on brachial artery blood flow before and following a resistance exercise bout.

Methods: In this, double-blinded placebo-controlled trial, college-aged males $(n=18)$ who self-reported recreationally resistance training for the previous $6 \pm 3$ years were assigned to one of two studies including a low (300 mg serving) $(n=9)$ or high dose (600 mg serving) ( $n=8 ; 1$ drop) GBS study. During testing sessions, participants reported to the laboratory following an overnight fast and rested in a supine position for 15 min. Thereafter, baseline measurements for resting heart rate (HR), systolic blood pressure (SBP), diastolic blood pressure (DBP), brachial artery diameter (BAD) and blood flow (BBF) were obtained (PRE). Participants then consumed either their respective GBS dose or a similar placebo dose (microcrystalline cellulose) in a supine resting state. HR, SBP, DBP, BAD and BBF were measured 45 min after placebo or GBS ingestion (PRE2). Participants were then placed in a recumbent position and performed 4 sets of 10 arm curl repetitions using an $11 \mathrm{~kg}$ dumbbell. Participants returned to a supine position and HR, SBP, DBP, BAD and BBF were obtained within the first 3 min following exercise (POST), $15 \mathrm{~min}$ after exercise (15POST), and $45 \mathrm{~min}$ after exercise (45POST). Participants returned to the laboratory 24-48 $\mathrm{h}$ later to repeat the same protocol with either GBS or the placebo depending on randomization. Two-way (supplement $x$ time) repeated measures ANOVAs were used to compare dependent variables between testing sessions for Study 1 (300 mg of GBS and placebo) and Study 2 (600 mg of GBS and placebo), and statistical significance was set at $p<0.05$. No statistical comparisons were made between studies.
\end{abstract}

(Continued on next page)

\footnotetext{
* Correspondence: mdr0024@auburn.edu

${ }^{1}$ Molecular and Applied Sciences Laboratory, School of Kinesiology, Auburn

University, 301 Wire Road, Office 286, Auburn, AL 36849, USA

${ }^{3}$ Edward Via College of Osteopathic Medicine Auburn, Auburn, AL, USA

Full list of author information is available at the end of the article
}

(c) The Author(s). 2020 Open Access This article is licensed under a Creative Commons Attribution 4.0 International License, which permits use, sharing, adaptation, distribution and reproduction in any medium or format, as long as you give appropriate credit to the original author(s) and the source, provide a link to the Creative Commons licence, and indicate if changes were made. The images or other third party material in this article are included in the article's Creative Commons licence, unless indicated otherwise in a credit line to the material. If material is not included in the article's Creative Commons licence and your intended use is not permitted by statutory regulation or exceeds the permitted use, you will need to obtain permission directly from the copyright holder. To view a copy of this licence, visit http://creativecommons.org/licenses/by/4.0/ The Creative Commons Public Domain Dedication waiver (http://creativecommons.org/publicdomain/zero/1.0/) applies to the data made available in this article, unless otherwise stated in a credit line to the data. 
(Continued from previous page)

Results: As expected, exercise increased BAD and BBF compared to resting baseline measured irrespective of supplementation. In addition, BAD and BBF did not differ between GBS and placebo at any time point after exercise in Study 1. In study 2, however, $600 \mathrm{mg}$ GBS increased baseline-normalized BBF at immediately post exercise compared to placebo (placebo $=211 \pm 155 \%$ increase, GBS $=349 \pm 156 \%$ increase; $p=0.012$ ) but not BAD.

Conclusions: These data suggest a higher dose of GBS can enhance localized blood flow acutely following a resistance exercise bout. However, the long-term implications of these data are unclear, and more well-powered studies are needed to validate efficacy and elucidate potential mechanisms.

Keywords: Blood flow, Green tea, Resistance exercise

\section{Background}

Nutritional supplements or dietary ingredients which promote vasodilation may be beneficial for increasing blood flow to working muscle during and following exercise (reviewed in $[1,2]$ ). Common dietary supplements that have been examined in this regard have included high nitratecontaining beet root juice [3-8], L-arginine [9], L-citrulline [10-12], betalains [13], and various plant extracts (e.g., red spinach) [14]. Most of these supplements have been posited to act through increasing endothelium-dependent nitric oxide (NOx) production which, in turn, stimulates smaller artery and arteriole vasodilation. However, some supplements (e.g., betalains) appear to enhance blood flow through NOx-independent mechanisms [13]. Additionally, while numerous independent laboratories have reported beet root juice increases humoral NOx levels and vasodilation, other supplements (e.g., L-arginine and L-citrulline) have not shown consistent results (reviewed in [2]).

While less-studied in humans, there is a body of evidence suggesting green tea catechins may also act as a putative blood flow-enhancing supplement. For instance, Potenza et al. [15] reported epigallocatechin gallate (EGCG) stimulates the formation of NOx and reduces blood pressure in spontaneously hypertensive rats, and similar results have been reported in other rodent studies $[16,17]$. However, unlike several of the aforementioned supplements, the effects of green tea supplements on peri-exercise blood flow patterns in humans have not been examined. Although the enhancement of periexercise blood flow does not have strong evidence to support ergogenecity, there is evidence to suggest enhanced blood flow supports certain phenotypic outcomes. For instance, older individuals with enhanced microvasculature properties may experience greater muscle growth during periods of resistance training [18]. Likewise, others have shown that an age-associated impairment in endothelial function reduces the anabolic response to amino acid feeding [19]. While these data are limited to older populations, these findings warrant future research into whether the enhancement of periexercise blood flow in proximity to a resistance training bout may provide certain anabolic benefits. There is also a plethora of literature to support the use of "blood flow enhancing" supplements to increase work and power output during submaximal and maximal exercise efforts. While mechanisms are currently lacking, it seems that enhanced blood flow through these supplements act to improve exercise economy (i.e., the amount of work able to be performed divided by oxygen consumption) (reviewed in [1, 20]. Thus, there is an interest in determining whether nutritional supplements augment periexercise blood flow.

The purpose of this study was to determine if lower (300 mg serving) or higher doses (600 mg serving) of a green tea-based supplement (GBS) affects brachial artery blood flow in college-aged males prior to and following acute resistance exercise compared to a placebo supplement. Given the previously mentioned animal studies, we hypothesized that lower and higher doses of GBS would enhance blood flow prior to and following exercise versus a placebo comparator.

\section{Methods \\ Participants}

This randomized double-blinded placebo controlled investigation was approved by the Institutional Review Board at Auburn University (Protocol\#19-162 AR 1905). Participants were included if they were recreationally active resistance trainers and free of cardiometabolic diseases (e.g., morbid obesity, type II diabetes, severe hypertension) or conditions which precluded exercise participation. Additionally, participants could not have consumed green tea-containing supplements, or other putative blood flow-enhancing supplements within one-month prior to participation. All participants provided verbal and written consent to participate, and this study conformed to the standards set by the latest revision of the Declaration of Helsinki.

\section{Experimental design}

In order to assess the efficacy of GBS on peri-exercise blood flow, we opted to examine two doses (300 mg and $600 \mathrm{mg}$ ); for the sake of clarity, we have termed these aims as "Study 1" and "Study 2" here in the methods. 
The first 9 participants that enrolled in the study were placed in Study 1 which examined $300 \mathrm{mg}$ of GBS (Vaso6, Compound Solutions, Carlsbad, CA, USA) versus $300 \mathrm{mg}$ placebo (microcrystalline cellulose, Compound Solutions). The remaining participants were placed in Study 2 which examined $600 \mathrm{mg}$ of GBS versus $600 \mathrm{mg}$ placebo.

All participants completed two workout visits which are described in greater detail below. During visit \#1 in both Study 1 and Study 2, participants were randomized to consume GBS or placebo using a coin flip method where one side of the coin indicated participants were to consume "A" pills, and the other side of the coin participants were to consume "B" pills. After completion of the first testing session, participants were required to return to the laboratory within $24-48 \mathrm{~h}$ for a second testing session to repeat these tests with either the placebo or GBS depending the coin flip randomization mentioned above. Critically, both the investigators and participants were blinded regarding which supplement as "A" versus "B". Likewise, supplements were in pill form and were undistinguishable to all parties involved.

The remainder of the methods describes the testing sessions as well as the experimental procedures used throughout the study.

\section{Testing sessions}

To control for the effects of diet and circadian patterns that affect hemodynamics, all participants reported to testing sessions during the morning hours (0700-1000) following an overnight fast. Additionally, participants were asked participants to refrain from all forms of structured resistance or endurance exercise the day prior to visit \#1 as well as between visits \#1 and \#2.

We adopted a study design similar to one that our laboratory has used in the past to examine the blood flowenhancing efficacy of an L-citrulline-containing nutritional supplement [10]. The main difference between studies is that we previously employed a leg extensor exercise bout and examined femoral artery blood flow patterns, whereas herein participants performed arm curl exercises and we examined brachial artery blood flow patterns. Notably, lower-body work with ultrasound has technical challenges given that: a) assessing the femoral artery can be intrusive with probe placement, and b) if calf extensions were allocated, consistently probing the popliteal artery is a technical challenge. Conversely, the brachial artery is relatively easy to probe, and the vessel is responsive to upper body exercise. Thus, this approach was adopted for the current study. Specific testing procedures are described in greater detail below.

\section{Bioelectrical impedance spectroscopy for whole-body composition assessment (first visit only)}

Body mass and composition was measured by bioelectrical impedance spectroscopy (BIS) using the SOZO device (ImpediMed Limited, Queensland, AU) according to the methods described by Moon et al. [21]. These methods have been shown to produce test-retest intraclass correlation coefficients (ICC) $>0.990$ for whole body intracellular and extracellular water metrics on 24 participants [22], and these devices estimate body fat percentage based on these metrics. Notably, body composition assessments were only used for phenotyping purposes, and were not a primary outcome measure.

Following body composition testing, participants were positioned on an athletic training table in a supine position for 15 min to allow for resting hemodynamic measures to be obtained. Thereafter, resting heart rate, systolic blood pressure (SBP), diastolic blood pressure (DBP), brachial artery diameter and blood flow were obtained as pre measures (PRE). Participants consumed either $300 \mathrm{mg}$ of GBS or placebo with $50 \mathrm{~mL}$ of tap water (Study 1, n=9), or $600 \mathrm{mg}$ of GBS or placebo with 50 $\mathrm{mL}$ of tap water (Study $2, n=9$ with 1 drop).

Heart rate, SBP, DBP, brachial artery diameter and blood flow were obtained $45 \mathrm{~min}$ following supplement ingestion (PRE2). Participants were placed in a recumbent position and performed 4 sets of 10 arm curl repetitions using an $11 \mathrm{~kg}$ dumbbell. Participants then returned to a supine position and heart rate, SBP, DBP, brachial artery diameter and blood flow were obtained within a 3 -min post exercise window (POST), $15 \mathrm{~min}$ following exercise (15POST), and $45 \mathrm{~min}$ following exercise (45POST).

Testing procedures for each of these measures are described in further detail below.

\section{Ultrasound for brachial artery blood flow assessment}

Blood flow through the right brachial artery was assessed using high resolution ultrasound (Logiq S7 R2 Expert; General Electric, Fairfield, CT, USA) with a 3 to $12 \mathrm{MHz}$ multi-frequency linear phase array transducer. The brachial artery was imaged longitudinally $8-10 \mathrm{~cm}$ distal from the shoulder joint. Simultaneous measurement of artery diameter and blood velocity was performed using duplex mode imaging (B-mode and Doppler) and video was captured through a digital interface at 30 frames/s with real time analysis (FMD Studio, Pisa, Italy). Measurements were captured for 2 continuous minutes with the transducer held by a supporting device in the same position. Vessel diameters were determined frame-byframe via automatic edge detection software (FMD Studio, Pisa, Italy) measuring the distance between the near and far wall of the intima. Blood velocity was determined via selection of a region of interest around the Doppler waveform and a trace of the velocity-time integral was used to calculate mean velocity for each cardiac cycle. Blood flow from continuous diameter and mean blood velocity measurements during ultrasonography were 
calculated as $\left[\Pi *(\text { diameter } / 2)^{2} *\right.$ time average mean velocity $* 120$ s].

\section{Heart rate and blood pressure determination}

Heart rate and blood pressure determinations occurred on the left arm simultaneously as right arm brachial artery blood flow data was being obtained using an automated blood pressure cuff (OMRON BP785, Omron Corporation, Kyoto, Japan). Triplicate values were averaged to obtain final values. The average time needed to obtain triplicate values was approximately $60-90 \mathrm{~s}$.

\section{Acute arm curl exercise bout}

Following PRE2 assessments, all participants were seated in a chair and performed 4 sets of 10 repetitions using a 25 -pound $(11 \mathrm{~kg})$ dumbbell. Participants were allowed 3 min of recovery between sets, and participants returned to the athletic training table and reclined in a supine position immediately following the fourth set for POST, 15POST and 45POST assessments. Given that the POST assessments occurred in closest proximity to exercise, a brief description will be provided on that time point. In short, it took approximately $8-10 \mathrm{~s}$ for participants to return to the athletic training table in a supine position. During the first minute following exercise, blood pressure and heart rate assessments began on the left arm, and triplicate measurements were completed by the second minute following exercise. The right arm brachial artery was located within the first minute following exercise, and data were obtained 1-3 min post-exercise. Thus, while these variables are described as "POST" throughout, the reader should be aware of the study mechanics described here.

\section{Statistics}

Two-way (supplement $\mathrm{x}$ time) repeated measures ANOVAs were used to compare dependent variables between testing sessions for Study 1 (300 mg of GBS and placebo) and Study 2 (600 mg of GBS and placebo), and statistical significance was set at $p<0.05$. No statistical comparisons were made between studies. In cases where Mauchly's test of sphericity indicated that data were not normally-distributed over time, Huynh-Feldt correction factors were applied to the data. LSD post hoc testing was performed when a significant supplement $\mathrm{x}$ time interaction was observed to determine which time points differed for a dependent variable between the placebo and GBS conditions. All data herein are presented in figures and tables as means \pm standard deviation values, and raw data are available in Supplemental File 1.

\section{Results}

Participant characteristics

Participant characteristics are presented in Table 1. Although 18 participants completed the protocol, one participant was
Table 1 Participant characteristics

\begin{tabular}{ll}
\hline Variable & Mean \pm SD \\
\hline Low GBS dose $(300 \mathrm{mg})$ experiment $(n=9)$ & $24 \pm 4$ \\
Age (years) & $83.0 \pm 9.4$ \\
Body mass $(\mathrm{kg})$ & $22.6 \pm 5.6$ \\
Body fat $(\%)$ & \\
High GBS dose $(600 \mathrm{mg})$ experiment $(n=8)$ & $26 \pm 4$ \\
Age (years) & $82.7 \pm 10.0$ \\
Body mass $(\mathrm{kg})$ & $18.1 \pm 3.8$ \\
Body fat $(\%)$ & Legend: All data are presented as means \pm standard deviation (SD) values
\end{tabular}

dropped from the analysis because his blood flow response to exercise was $\sim 2$-fold greater than all other participants, and this response was $>3$ standard deviations from the mean response. Of the remaining 17 participants, all but one participant (16 of 17) self-reported being recreationally resistance-trained $6 \pm 4 \mathrm{~h}$ per week for $6 \pm 3$ years.

\section{Heart rate and blood pressure responses}

Heart rate and blood pressure responses to the low and high dose GBS experiments are presented in Table 2. In the lower-dose experiment there was significant time effect for heart rate $(p=0.005)$, and post hoc testing indicated that this variable significantly decreased at PRE2 and 45POST relative to PRE $(p<0.05)$. There was also a significant time effect for SBP $(p=0.001)$, and post hoc testing indicated that this variable significantly increased at POST relative to PRE $(p<0.05)$. However, there were no supplement $x$ time effects for any of these variables.

In the higher-dose experiment there was significant time effect for heart rate $(p=0.027)$, and post hoc testing indicated that this variable significantly decreased at 45POST relative to PRE $(p<0.05)$. There was also a significant time effect for SBP $(p<0.001)$, and post hoc testing indicated that this variable significantly increased at POST relative to PRE $(p<0.05)$. However, there were no supplement $x$ time effects for any of these variables.

\section{Blood flow and brachial artery diameter responses}

Blood flow and brachial artery diameter responses to the lower- and higher-dose GBS experiments are presented in Fig. 1. In the lower-dose experiment there were significant time effects for blood flow $(p<0.001)$ (Fig. 1a) and brachial artery diameter $(p<0.001)$ (Fig. 1b). Post hoc testing indicated that blood flow significantly increased at POST relative to PRE $(p<0.001)$, and diameter increased at PRE2 thru 45POST relative to PRE $(p<0.05)$. However, there were no supplement $x$ time effects for these variables.

In the higher-dose experiment there was a significant time effect for blood flow ( $p=0.001)$ (Fig. 1c). Post hoc testing indicated that blood flow significantly increased at POST relative to PRE $(p<0.001)$ and $45 \mathrm{POST}$ was 
Table 2 Heart rate and blood pressure responses

\begin{tabular}{|c|c|c|c|c|c|c|}
\hline Time point & PRE & PRE2 & POST & 15POST & 45POST & Statistics \\
\hline \multicolumn{7}{|c|}{ Low GBS dose $(300 \mathrm{mg})(n=9)$} \\
\hline Heart rate (bpm) & & $\#$ & & & $\#$ & Supp $p=0.702$ \\
\hline GBS & $62 \pm 12$ & $59 \pm 10$ & $60 \pm 10$ & $62 \pm 12$ & $60 \pm 10$ & Time $p=0.005$ \\
\hline Placebo & $61 \pm 13$ & $57 \pm 12$ & $60 \pm 11$ & $58 \pm 11$ & $58 \pm 11$ & $\mathrm{Sx} T p=0.669$ \\
\hline $\mathrm{SBP}(\mathrm{mmHg})$ & & & * & & & Supp $p=0.924$ \\
\hline GBS & $115 \pm 8$ & $115 \pm 7$ & $119 \pm 9$ & $114 \pm 9$ & $114 \pm 8$ & Time $p=0.001$ \\
\hline Placebo & $115 \pm 8$ & $114 \pm 7$ & $120 \pm 7$ & $113 \pm 5$ & $116 \pm 7$ & $\mathrm{Sx} T p=0.867$ \\
\hline $\mathrm{DBP}(\mathrm{mmHg})$ & & & & & & Supp $p=0.516$ \\
\hline GBS & $64 \pm 7$ & $63 \pm 7$ & $63 \pm 7$ & $63 \pm 6$ & $62 \pm 6$ & Time $p=0.939$ \\
\hline Placebo & $63 \pm 6$ & $65 \pm 6$ & $65 \pm 5$ & $64 \pm 6$ & $66 \pm 6$ & $\mathrm{Sx} \top p=0.475$ \\
\hline \multicolumn{7}{|c|}{ High GBS dose $(600 \mathrm{mg})(n=8)$} \\
\hline Heart rate (bpm) & & & & & $\#$ & Supp $p=0.825$ \\
\hline GBS & $56 \pm 8$ & $55 \pm 10$ & $56 \pm 11$ & $55 \pm 9$ & $54 \pm 7$ & Time $p=0.027$ \\
\hline Placebo & $56 \pm 8$ & $54 \pm 9$ & $56 \pm 8$ & $56 \pm 8$ & $54 \pm 8$ & SxT $p=0.473$ \\
\hline $\mathrm{SBP}(\mathrm{mmHg})$ & & & * & & & Supp $p=0.341$ \\
\hline GBS & $112 \pm 6$ & $113 \pm 6$ & $120 \pm 7$ & $113 \pm 7$ & $114 \pm 7$ & Time $p<0.001$ \\
\hline Placebo & $116 \pm 4$ & $114 \pm 4$ & $120 \pm 4$ & $116 \pm 4$ & $115 \pm 3$ & $\mathrm{Sx} T p=0.239$ \\
\hline $\mathrm{DBP}(\mathrm{mmHg})$ & & & & & & Supp $p=0.857$ \\
\hline GBS & $64 \pm 5$ & $66 \pm 5$ & $67 \pm 5$ & $67 \pm 6$ & $68 \pm 6$ & Time $p=0.454$ \\
\hline Placebo & $67 \pm 7$ & $66 \pm 7$ & $66 \pm 5$ & $67 \pm 4$ & $66 \pm 6$ & SxT $p=0.397$ \\
\hline
\end{tabular}

Legend: All hemodynamic data are presented as mean \pm SD. Abbreviations: bpm Beats per minute, $\mathrm{mmHg}$ Millimeters of mercury, SBP Systolic blood pressure, $D B P$ Diastolic blood pressure, PRE Pre-supplement consumption; PRE2 45-min post-supplement consumption, POST within 3 min of arm curl exercise (4 sets of 10 repetitions using $11 \mathrm{~kg}$ ), 15 POST $15 \mathrm{~min}$ post-exercise, 45POST 45 min post-exercise, Supp main effect of supplement (GBS versus placebo), Time main effect of time, SXT supplement by time interaction. Symbols: *, time effect post hoc indicated greater than PRE $(p<0.05)$; \#, time effect post hoc indicated lower than PRE $(p<0.05)$

lower than PRE $(p<0.05)$. The supplement $\mathrm{x}$ time interaction also approached significance $(p=0.086)$, and post hoc testing indicated GBS trended higher than placebo at POST $(p=0.076)$.

There was a significant time effect for brachial artery diameter $(p<0.001)$ (Fig. 1d). Post hoc testing indicated diameter increased at POST and 15POST relative to PRE $(p<0.05)$. However, there were no supplement $\mathrm{x}$ time effects for this variable.

Given the blood flow trend for the higher dose GBS experiment, we normalized blood flow as percent change from the PRE testing point value in a post hoc fashion in order to derive blood flow changes relative to presupplement ingestion (Fig. 2). Notably, this approach has been used prior in order to reduce the between-subject variability in baseline blood flow measurements prior to an experimental treatment [23]. Again, the supplement $x$ time interaction approached significance $(p=0.086)$, and post hoc testing indicated $600 \mathrm{mg}$ GBS was significantly greater than $600 \mathrm{mg}$ placebo at POST $(p=0.012)$.

\section{Discussion}

There is ample evidence suggesting select nutritional supplements are capable of enhancing peri-exercise blood flow and, as mentioned prior, numerous rodent studies have reported that green tea catechins are capable of promoting vasodilation [15-17]. However, this is the first study demonstrating that a green tea-based supplement can enhance localized blood flow in humans following a resistance exercise bout.

While these data are promising, there are still several questions that remain to be answered. First, it is unclear why $600 \mathrm{mg}$ GBS was effective at increasing postexercise blood flow, whereas $300 \mathrm{mg}$ was not. While human data are lacking, it is notable that researchers have shown EGCG to have a dose-dependent effect on rodent hind limb microvascular vasodilation [24]. Thus, our data imply that (on average) a $600 \mathrm{mg}$ dose may be efficacious at eliciting an increase in post-exercise blood flow in humans, whereas lower doses are not as effective. It is also curious that superior post-exercise blood flow increases during the $600 \mathrm{mg}$ GBS versus placebo condition were observed without an increase in brachial artery diameter. Physiological variables that affect blood flow include vessel diameter, vessel length, blood viscosity, heart rate, and blood pressure [25]. It is logical to assume that the acute ingestion of GBS did not affect vessel length and likely did not affect blood hematocrit 

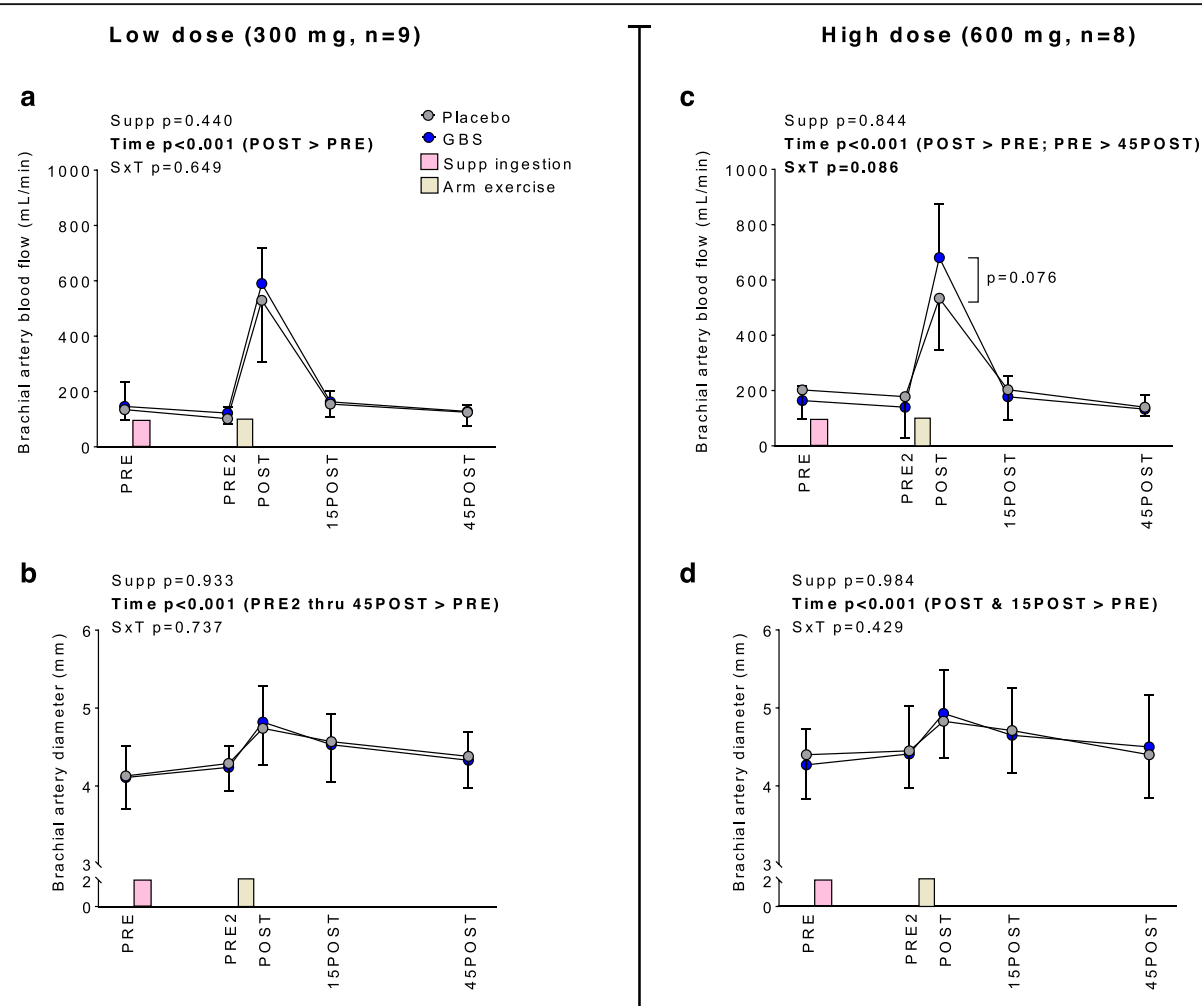

Fig. 1 Blood flow and brachial artery responses to lower and higher doses of GBS. Legend: The effects of lower and higher doses of GBS on brachial artery blood flow (panels $\mathbf{a}$ and $\mathbf{c}$, respectively) and brachial artery diameter (panels $\mathbf{b}$ and $\mathbf{d}$ respectively). All data are presented as means \pm SD values. Abbreviations: PRE, pre-supplement consumption; PRE2, 45-min post-supplement consumption; POST, within 5 min of arm curl exercise (4 sets of 10 repetitions using $11 \mathrm{~kg}$ ); 15POST, 15 min post-exercise; 45POST, 45 min post-exercise; Supp, main effect of supplement (GBS versus placebo); Time, main effect of time; SxT, supplement by time interaction

levels or blood viscosity, although we did not assay for the latter two variables. We also posit that GBS-induced increases in blood flow were not due to increased heart rate or blood pressure given that these readings were similar between the $600 \mathrm{mg}$ GBS and placebo conditions. However, it is possible that higher-dose GBS ingestion enhanced the vasodilation of smaller arterioles downstream of the brachial artery which, in turn, decreased peripheral resistance to blood flow allowing for increased velocities. Given that we currently lack these data, future studies implementing the current study design and using small vessel monitoring techniques (e.g., NIRS [26] or stain-gauge plethysmography [27]) are needed to validate this hypothesis. It is also notable that humoral signaling mediators (e.g., prostaglandins) as well as localized factors (e.g., smooth muscle NOx and potassium concentrations) affect blood flow [28]. Thus, it is possible that higher doses of GBS may operate through these mediators, and more research is needed in this regard.

Although $600 \mathrm{mg}$ of GBS transiently increased postexercise blood flow, it is currently unclear how this phenomena would affect resistance training adaptations. As mentioned prior, recent research in older populations has suggested peri-exercise blood flow may positively impact the anabolic response to feeding and resistance training. Critically, the current study does not guarantee or even insinuate that GBS supplementation during resistance training would enhance muscle mass gains. In fact, the nutritional supplement arginine, which is known to stimulate vasodilation, has been shown not to affect muscle mass changes in men that resistance trained [29]. Notwithstanding, arginine and other supplements that increase per-exercise blood flow (e.g., Lcitrulline and beet root juice and extracts) have been found to prolong work and/or power output during maximal resistance exercise repetition attempts, sprinting efforts, and submaximal aerobic exercise events [10, 12, 13, 29-33]. Therefore, future research should be performed to determine if the GBS-induced increase in peri-exercise blood flow is associated with any of these potential ergogenic effects.

There are certain limitations to this study. First, we lack mechanistic data as mentioned above, and it is currently unclear why $600 \mathrm{mg}$ GBS did not affect blood flow prior to exercise. Indeed, we have observed a similar 


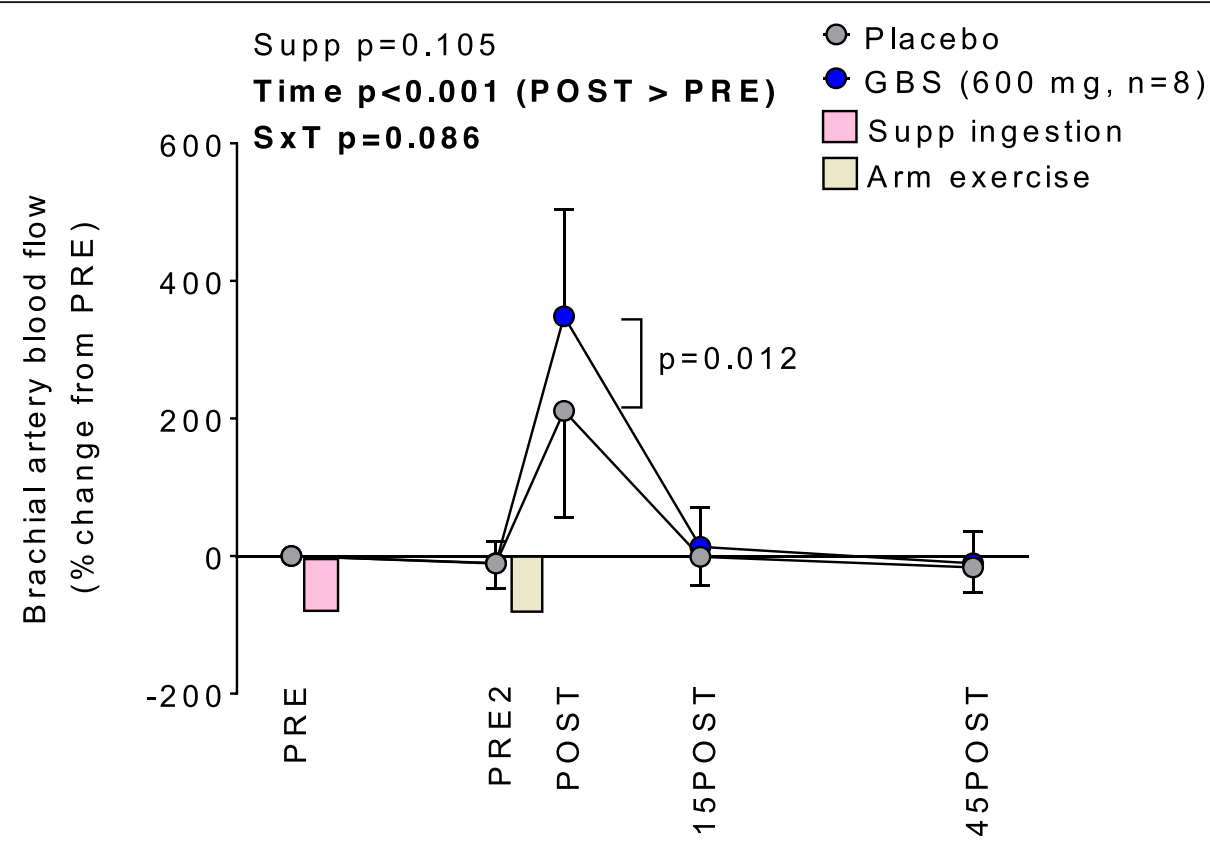

Fig. 2 Blood flow normalized to PRE values during the higher GBS dosage experiment. Legend: The effects of higher doses of GBS on brachial artery blood flow normalized to PRE values. Data are presented as means \pm SD values. Abbreviations: PRE, pre-supplement consumption; PRE2, 45min post-supplement consumption; POST, within 5 min of arm curl exercise (4 sets of 10 repetitions using 11 kg); 15 POST, 15 min post-exercise; 45POST, 45 min post-exercise; Supp, main effect of supplement (GBS versus placebo); Time, main effect of time; SxT, supplement by time interaction

phenomenon with a supplement containing L-citrulline. Specifically, compared to a placebo condition, femoral artery blood flow was not affected up to 45 min following supplement ingestion and prior to a leg extensor resistance training bout, but was significantly greater immediately post-exercise [10]. Unlike nitrate-containing supplements, it is possible that some "blood-flow enhancing" nutritional supplements may act, in part, to potentiate localized factors released from skeletal muscle or endothelial cells to enhance blood flow. To this end, more mechanistic data are needed to validate this hypothesis. This study was also limited to n-sizes of 8-9 participants in the lower- and higherdose studies due to resource constraints. Hence, more wellpowered studies (i.e., 20-30 participants with a crossover design based upon sample size calculations using the current data) are needed to validate the efficacy of GBS, and elucidate potential mechanisms through which blood flow is enhanced. Additionally, it is currently unknown if higher doses (e.g., 900 or $1200 \mathrm{mg}$ ) have additive effects on enhancing blood flow and/or brachial artery diameters, and this warrants further consideration. Finally, it is notable that only a standardized dumbbell $(11 \mathrm{~kg})$ was used for the exercise bout for all participants. Our reasoning for this was that, unlike other exercises such as the leg press or back squat, our laboratory has found it difficult in the past to assess a one-repetition maximum using a single-arm curl exercise. Thus, we were against attempting to prescribe the arm curl exercise bout based upon a predetermined relative strength, and instead posited that $11 \mathrm{~kg}$ is a weight that is commonly used by recreational lifters for higher-volume sets in the gymnasium.

\section{Conclusions}

This is the first evidence suggesting a green tea-based supplement enhances localized blood flow following a resistance exercise bout. Indeed, more definitive evidence is needed to validate mechanisms, and the longterm implications of these findings for individuals who habitually resistance train are unknown and require further investigation.

\section{Supplementary information}

Supplementary information accompanies this paper at https://doi.org/10. 1186/s12970-020-00358-5.

\section{Additional file 1}

\section{Abbreviations}

BAD: Brachial artery diameter; BBF: Brachial artery blood flow; DBP: Diastolic blood pressure; HR: Heart rate; SBP: Systolic blood pressure

\section{Acknowledgements}

The authors would like to sincerely thank all participants for their dedicated effort throughout the investigation and willingness to participate.

\section{Authors' contributions}

This experiment was performed at Auburn University's School of Kinesiology in the Molecular and Applied Sciences Laboratory and Applied Physiology Laboratory. MDR was responsible for the conception and design of the 
experiment, and primarily drafted the manuscript. CDF performed all testing procedures. All other co-authors were involved with data collection, and read and approved the final manuscript.

\section{Funding}

Funding for subject compensation were provided by donations in kind to MDR from Emerge Sales (Patchogue, NY, USA).

\section{Availability of data and materials}

All raw data are available in Supplemental File 1.

\section{Ethics approval and consent to participate}

All procedures described herein were approved by the Auburn University Institutional Review Board (Protocol\#19-162 AR 1905) and conformed to the standards set by the latest revision of the Declaration of Helsinki. All subjects provided written and verbal consent prior to study participation.

\section{Consent for publication}

Not applicable.

\section{Competing interests}

The authors declare that they have no competing interests.

\section{Author details}

'Molecular and Applied Sciences Laboratory, School of Kinesiology, Auburn University, 301 Wire Road, Office 286, Auburn, AL 36849, USA. ${ }^{2}$ Exercise and Performance Nutrition Laboratory, School of Health Sciences, Lindenwood University, Saint Charles, MO, USA. ${ }^{3}$ Edward Via College of Osteopathic Medicine Auburn, Auburn, AL, USA.

Received: 8 January 2020 Accepted: 19 May 2020

Published online: 27 May 2020

\section{References}

1. Harty PS, Zabriskie HA, Erickson JL, Molling PE, Kerksick CM, Jagim AR. Multiingredient pre-workout supplements, safety implications, and performance outcomes: a brief review. J Int Soc Sports Nutr. 2018;15(1):41.

2. Kerksick CM, Wilborn CD, Roberts MD, Smith-Ryan A, Kleiner SM, Jager R, et al. ISSN exercise \& sports nutrition review update: research \& recommendations. J Int Soc Sports Nutr. 2018;15(1):38.

3. Kenjale AA, Ham KL, Stabler T, Robbins JL, Johnson JL, Vanbruggen M, et al. Dietary nitrate supplementation enhances exercise performance in peripheral arterial disease. J Appl Physiol (1985). 2011;110(6):1582-91.

4. Breese BC, McNarry MA, Marwood S, Blackwell JR, Bailey SJ, Jones AM. Beetroot juice supplementation speeds $\mathrm{O} 2$ uptake kinetics and improves exercise tolerance during severe-intensity exercise initiated from an elevated metabolic rate. Am J Phys Regul Integr Comp Phys. 2013;305(12): R1441-50.

5. Vanhatalo A, Fulford J, Bailey SJ, Blackwell JR, Winyard PG, Jones AM. Dietary nitrate reduces muscle metabolic perturbation and improves exercise tolerance in hypoxia. J Physiol. 2011;589(Pt 22):5517-28.

6. Vanhatalo A, Jones AM, Blackwell JR, Winyard PG, Fulford J. Dietary nitrate accelerates postexercise muscle metabolic recovery and $\mathrm{O} 2$ delivery in hypoxia. J Appl Physiol (1985). 2014;117(12):1460-70.

7. Lansley KE, Winyard PG, Fulford J, Vanhatalo A, Bailey SJ, Blackwell JR, et al. Dietary nitrate supplementation reduces the $\mathrm{O} 2$ cost of walking and running: a placebo-controlled study. J Appl Physiol (1985). 2011;110(3):591600.

8. Bailey SJ, Varnham RL, DiMenna FJ, Breese BC, Wylie LJ, Jones AM. Inorganic nitrate supplementation improves muscle oxygenation, O (2) uptake kinetics, and exercise tolerance at high but not low pedal rates. J Appl Physiol (1985). 2015:118(11):1396-405.

9. Mehta S, Stewart DJ, Levy RD. The hypotensive effect of L-arginine is associated with increased expired nitric oxide in humans. Chest. 1996;109(6): 1550-5.

10. Martin JS, Mumford PW, Haun CT, Luera MJ, Muddle TWD, Colquhoun RJ, et al. Effects of a pre-workout supplement on hyperemia following leg extension resistance exercise to failure with different resistance loads. J Int Soc Sports Nutr. 2017;14:38.
11. Hickner RC, Tanner CJ, Evans CA, Clark PD, Haddock A, Fortune C, et al. Lcitrulline reduces time to exhaustion and insulin response to a graded exercise test. Med Sci Sports Exerc. 2006;38(4):660-6.

12. Suzuki T, Morita M, Kobayashi $Y$, Kamimura A. Oral L-citrulline supplementation enhances cycling time trial performance in healthy trained men: double-blind randomized placebo-controlled 2-way crossover study. J Int Soc Sports Nutr. 2016;13:6.

13. Mumford PW, Kephart WC, Romero MA, Haun CT, Mobley CB, Osburn SC, et al. Effect of 1-week betalain-rich beetroot concentrate supplementation on cycling performance and select physiological parameters. Eur J Appl Physiol. 2018;118(11):2465-76.

14. Haun CT, Kephart WC, Holland AM, Mobley CB, McCloskey AE, Shake JJ, et al. Differential vascular reactivity responses acutely following ingestion of a nitrate rich red spinach extract. Eur J Appl Physiol. 2016;116(11-12):226779.

15. Potenza MA, Marasciulo FL, Tarquinio M, Tiravanti E, Colantuono G, Federici A, et al. EGCG, a green tea polyphenol, improves endothelial function and insulin sensitivity, reduces blood pressure, and protects against myocardial I/R injury in SHR. Am J Physiol Endocrinol Metab. 2007;292(5):E1378-87.

16. Kim JA, Formoso G, Li Y, Potenza MA, Marasciulo FL, Montagnani M, et al. Epigallocatechin gallate, a green tea polyphenol, mediates NO-dependent vasodilation using signaling pathways in vascular endothelium requiring reactive oxygen species and Fyn. J Biol Chem. 2007:282(18):13736-45.

17. Lorenz M, Wessler S, Follmann E, Michaelis W, Dusterhoft T, Baumann G, et al. A constituent of green tea, epigallocatechin-3-gallate, activates endothelial nitric oxide synthase by a phosphatidylinositol-3-OH-kinase-, CAMP-dependent protein kinase-, and Akt-dependent pathway and leads to endothelial-dependent vasorelaxation. J Biol Chem. 2004;279(7):6190-5.

18. Snijders T, Nederveen JP, Joanisse S, Leenders M, Verdijk LB, van Loon LJ, et al. Muscle fibre capillarization is a critical factor in muscle fibre hypertrophy during resistance exercise training in older men. J Cachexia Sarcopenia Muscle. 2017;8(2):267-76.

19. Timmerman KL, Dhanani S, Glynn EL, Fry CS, Drummond MJ, Jennings K, et al. A moderate acute increase in physical activity enhances nutritive flow and the muscle protein anabolic response to mixed nutrient intake in older adults. Am J Clin Nutr. 2012;95(6):1403-12.

20. Dominguez R, Cuenca E, Mate-Munoz UL, Garcia-Fernandez P, Serra-Paya N, Estevan MC, et al. Effects of Beetroot Juice Supplementation on Cardiorespiratory Endurance in Athletes. A Systematic Review. Nutrients. 2017:9(1):43.

21. Moon JR, Tobkin SE, Roberts MD, Dalbo VJ, Kerksick CM, Bemben MG, et al. Total body water estimations in healthy men and women using bioimpedance spectroscopy: a deuterium oxide comparison. Nutr Metab (Lond). 2008;5:7

22. Haun CT, Vann CG, Mobley CB, Roberson PA, Osburn SC, Holmes HM, et al. Effects of graded whey supplementation during extreme-volume resistance training. Front Nutr. 2018;5:84.

23. Hunter AM, Grigson C, Wade A. Influence of topically applied menthol cooling gel on soft tissue thermodynamics and arterial and cutaneous blood flow at rest. Int J Sports Phys Ther. 2018;13(3):483-92.

24. Ng HLH, Premilovac D, Rattigan S, Richards SM, Muniyappa R, Quon MJ, et al. Acute vascular and metabolic actions of the green tea polyphenol epigallocatechin 3-gallate in rat skeletal muscle. J Nutr Biochem. 2017;40: 23-31.

25. Braunwald E, Sarnoff SJ, Case RB, Stainsby WN, Welch GH Jr. Hemodynamic determinants of coronary flow: effect of changes in aortic pressure and cardiac output on the relationship between myocardial oxygen consumption and coronary flow. Am J Phys. 1958;192(1):157-63.

26. Jones S, Chiesa ST, Chaturvedi N, Hughes AD. Recent developments in nearinfrared spectroscopy (NIRS) for the assessment of local skeletal muscle microvascular function and capacity to utilise oxygen. Artery Res. 2016;16: 25-33.

27. Benjamin N, Calver A, Collier J, Robinson B, Vallance P, Webb D. Measuring forearm blood flow and interpreting the responses to drugs and mediators. Hypertension. 1995;25(5):918-23.

28. Joyner MJ, Casey DP. Regulation of increased blood flow (hyperemia) to muscles during exercise: a hierarchy of competing physiological needs. Physiol Rev. 2015;95(2):549-601.

29. Campbell B, Roberts M, Kerksick C, Wilborn C, Marcello B, Taylor L, et al. Pharmacokinetics, safety, and effects on exercise performance of $L$-arginine alpha-ketoglutarate in trained adult men. Nutrition. 2006;22(9):872-81. 
30. Suzuki I, Sakuraba K, Horiike T, Kishi T, Yabe J, Suzuki T, et al. A combination of oral $\mathrm{L}$-citrulline and $\mathrm{L}$-arginine improved 10-min full-power cycling test performance in male collegiate soccer players: a randomized crossover trial. Eur J Appl Physiol. 2019:119(5):1075-84.

31. Bailey SJ, Blackwell JR, Lord T, Vanhatalo A, Winyard PG, Jones AM. ICitrulline supplementation improves $\mathrm{O} 2$ uptake kinetics and high-intensity exercise performance in humans. J Appl Physiol (1985). 2015;119(4):385-95.

32. Cermak NM, Gibala MJ, van Loon LJ. Nitrate supplementation's improvement of 10-km time-trial performance in trained cyclists. Int J Sport Nutr Exerc Metab. 2012;22(1):64-71.

33. Jo E, Fischer M, Auslander AT, Beigarten A, Daggy B, Hansen $\mathrm{K}$, et al. The effects of multi-day vs. single pre-exercise nitrate supplement dosing on simulated cycling time trial performance and skeletal muscle oxygenation. J Strength Cond Res. 2019;33(1):217-24.

\section{Publisher's Note}

Springer Nature remains neutral with regard to jurisdictional claims in published maps and institutional affiliations.

Ready to submit your research? Choose BMC and benefit from:

- fast, convenient online submission

- thorough peer review by experienced researchers in your field

- rapid publication on acceptance

- support for research data, including large and complex data types

- gold Open Access which fosters wider collaboration and increased citations

- maximum visibility for your research: over $100 \mathrm{M}$ website views per year

At BMC, research is always in progress.

Learn more biomedcentral.com/submissions 